\title{
La accessibilidad de las webs de las universidades españolas. Balance 2001-2006
}

\author{
Mireia Ribera*, Miquel Térmens*, Amparo Frías*
}

\begin{abstract}
Resumen: El estudio analiza el nivel de cumplimiento que el año 2006 hacían las sedes web de las universidades españolas de las Pautas de accesibilidad al contenido de la web (WCAG), versión 1.0, en su nivel A. Los resultados del análisis se comparan con los obtenidos el año 2001. Por el lado positivo se observa una mayor implantación de las alternativas a gráficos, hojas de estilo CSS y de las alternativas a Flash y PDF; y que un número mayor de universidades (10 en total) logra en 2006 un cumplimiento al $100 \%$ del nivel A de las WCAG. Por el lado negativo se observa una peor calidad de los textos y un menor cumplimiento de las normas de accesibilidad, respecto a los resultados del año 2001, en las páginas de los planes de estudio, usualmente generadas de forma automática. El estudio, de forma complementaria, también analiza el nivel de seguimiento de los indicadores de la norma 508 de Estados Unidos y de otros 5 indicadores adicionales de usabilidad. En cuanto al cumplimiento de la norma 508, no obligatoria en España, ninguna universidad logra el 100\% de cumplimiento. Respecto a los otros indicadores el cumplimiento es de un $65 \%$. Como resultado global se constata que el nivel de accesibilidad sigue en niveles bajos, similar al del año 2001, y con grandes diferencias entre centros; estos resultados indican un nivel de cumplimiento menor del que cabría esperar por los cambios aplicados en la legislación española a favor de la accesibilidad de los sitios web.
\end{abstract}

Palabras clave: accesibilidad, usabilidad, WAI, WCAG, universidad española.

\section{The accessibility of Spanish university web sites. \\ A 2001-2006 assessment}

Abstract: This study examines the compliance of the web sites of Spanish universities with level $A$ of the Web Content Access Guidelines (WCAG) v. 1.0 in 2006, and compares the obtained results with those of 2001. On the positive side, in 2006 more use of alternatives to graphics was made, cascading style sheets and the use of Flash and PDF. In addition, more universities (10 in total) complied 100\% with the WCAG at level A. On the negative side, the texts were of poorer quality than in 2001, and fewer complied with accessibility standards with respect to plans of study, which were usually automatically generated. An analysis was also made of the compliance of indicators with American standard 508 (which is not mandatory in Spain) and five other usability indicators. No university complied 100\% with standard 508; compliance with the other indicators was $65 \%$. The results show that the level of accessibility remains low, similar

* Departament de Biblioteconomia i Documentació, Universitat de Barcelona. Correo-e: ribera@ ub.edu.termens@ub.edu, empar.frias@castellbisbal.cat.

Recibido: 1-10-08; 2. ${ }^{\mathrm{a}}$ versión: 11-1-09. 
in fact to the situation in 2001, but with large differences between universities. Indeed, the level of compliance is less than that which might be expected given the changes in Spanish law made to favour access to public web sites.

Keywords: accessibility, usability, WAI, WCAG, Spanish universities.

\section{Introducción}

El año 2001 los autores del presente artículo ya estudiamos el nivel de accesibilidad de las sedes web de las universidades españolas (Térmens, Ribera, Sulé, 2003). Comprobamos el nivel de cumplimiento en esas páginas de las directrices de las Pautas de accesibilidad al contenido de la web -WCAG_, versión 1.0 de mayo de 1999, publicadas por el World-Wide Web Consortium —W3C- (Web, 1999).

En ese momento las directrices WCAG no eran de obligado cumplimiento en España aunque, como ya se indicaba en aquel estudio, a nivel internacional se estaban adoptando rápidamente como norma obligatoria, al menos para las sedes web dependientes de la administración pública. Desde esa fecha, la situación legal ha cambiado bastante en España, en parte por requerimiento de la Unión Europea y en parte como resultado del despliegue normativo de la legislación de accesibilidad en España. En el transcurso de estos años también se ha constatado un claro avance en la sensibilización general respecto a la problemática de la accesibilidad electrónica. En este contexto, y transcurridos cinco años desde el estudio inicial, pareció oportuno ejecutar una nueva revisión de la accesibilidad de las sedes web de las universidades españolas. Con ella pretendemos medir cuál ha sido el impacto real, efectivo, de los cambios legales y del aumento de la sensibilización hacia la accesibilidad web. Estos resultados han de ser esclarecedores de la situación actual, en un momento en el que la última legislación española reglamenta que a partir de 31 de diciembre de 2008 todos los sitios web dependientes de las administraciones públicas han de ser accesibles no solo a un nivel "A", como el estudiado en el presente trabajo, sino en el nivel superior "AA". Recordemos que las directrices WCAG establecen tres niveles de cumplimiento —A, AA y AAA—, según el nivel creciente de soluciones de accesibilidad aplicadas, siendo A un nivel básico y AAA un nivel completo. La mayoría de gobiernos han optado por establecer en el nivel intermedio "AA" la obligatoriedad en sus respectivas legislaciones de accesibilidad en la web.

\section{Revisión bibliográfica}

Desde 2001 la evaluación de la accesibilidad de sitios web universitarios ha sido un campo activo de investigación con numerosas publicaciones; una buena recopilación se puede consultar en Web Accessibility Survey Site, mantenido por Axel Schmetzke, en http://library.uwsp.edu/aschmetz/Accessible/websurveys.htm. 
En España el Observatorio de la InfoAccesibilidad Digital ${ }^{1}$, dependiente de Discapnet, ha realizado diversos estudios sobre portales españoles, entre ellos destacamos La accesibilidad de los portales universitarios en España (Accesibilidad, 2004), con un planteamiento bastante paralelo al presente, aunque con una menor cobertura de centros y sin incorporar un análisis comentado. También se ha de mencionar el estudio realizado por la consultora Acctiva (Breve, 2004). Otros estudios han preferido realizar análisis más profundos, incluyendo aspectos de usabilidad, (García, 2006) aunque a veces centrándose en un número limitado de universidades (Pinto et al., 2005). Nuestro propio equipo también ha publicado estudios regionales (Térmens, Ribera, Sulé, 2002; Térmens, 2002).

Aunque muchos de los estudios siguen basándose exclusivamente en las WCAG la tendencia más actual es completar la evaluación de la accesibilidad con otras herramientas propias de la ingeniería de la usabilidad: test de usuarios, revisión de directrices diversas, encuestas o focus groups. En el plano teórico, Kelly y Sloan (Kelly, 2005) (Sloan, 2006) abogan por una implementación holística de las WCAG que tenga en cuenta el contexto, la audiencia, etc.; Mankoff (Mankoff, 2005) compara diversos métodos para evaluar la accesibilidad para personas ciegas y recomienda el uso de la técnica de screening con lectores de pantalla con mejores resultados que la aplicación de directrices; Milne (Milne, 2005) menciona las flaquezas de las WCAG para personas mayores y propone soluciones. A nivel aplicado, el estudio de la Disability Rights Commission (DRC, 2004) creó mucha polémica al cuestionar la validez de las WCAG como único método de evaluación. En la línea de estas visiones, compartidas por los autores, la investigación presentada se ofrece como una revisión diacrónica del estudio de 2001; los resultados también se han de entender como un simple indicador de la accesibilidad.

\section{El contexto español}

El cambio más importante para la accesibilidad web ha sido el despliegue normativo a nivel europeo y en España de los principios de accesibilidad universal. Este estudio pretende verificar la trascendencia de estos cambios legislativos en la práctica real del diseño accesible.

A nivel europeo, como parte integral del Plan de Acción para el 2002, de la Iniciativa eEurope, la Comisión Europea presentó el 25 de septiembre de 2001 la comunicación eEurope 2002: Accesibilidad de los sitios Web públicos y de su contenido ${ }^{2}$, que insistió en la exigencia de cumplir con los plazos marcados y, por tanto, en que los sitios web de la administración pública cumplieran a finales del año 2001 con el nivel "A" de las pautas WCAG.

\footnotetext{
${ }^{1}$ http://www.discapnet.es/Discapnet/Castellano/Observatorio_infoaccesibilidad/default.htm.

2 http://eur-lex.europa.eu/LexUriServ/LexUriServ.do?uri=COM:2001:0529:FIN:ES:PDF.
} 
El 14 de enero de 2003 el Consejo de la Unión Europea adoptó la resolución Mejorar el acceso de las personas con discapacidad a la sociedad del conocimien$t o^{3}$ que instaba a los estados miembros a llevar a cabo una serie de medidas para fomentar la accesibilidad electrónica. Posteriormente, en la directiva 2004/18/CE del Parlamento Europeo y del Consejo, de 31 de marzo de 2004, se establecían los criterios de accesibilidad en los procedimientos de adjudicación de los contratos públicos de obras, de suministro y de servicios; la directiva establecía el 31 de enero de 2006 como fecha límite para la adaptación de las distintas legislaciones nacionales ${ }^{4}$. En septiembre de 2005, se publicó una nueva comunicación COM(2005)425 de la Comisión eAccessibility, en la que se insistía en la necesidad de la accesibilidad electrónica 5 .

En España como primera ley importante cabe citar la Ley 34/2002, de 11 de julio, de servicios de la sociedad de la información y de comercio electrónico (LSSICE). En materia de accesibilidad dispone que las administraciones públicas y los servicios por ellas financiados deberán ser accesibles antes del 31 de diciembre de 2005, y que se promoverá la adopción de normas de accesibilidad a otros actores de la sociedad de la información.

Aún así, la Ley 51/2003, de 2 de diciembre, de igualdad de oportunidades, no discriminación y accesibilidad universal de las personas con discapacidad (LIONDAU), que no se desplegó totalmente hasta el año 2007, es la que concreta y estipula las medidas exactas en las que consistirá la promoción y obligaciones en cuanto a accesibilidad electrónica se refiere. La LIONDAU establece que las páginas web se deberán adaptar a la normativa de accesibilidad vigente y comúnmente aceptada, es decir las WCAG. En la legislación española una ley no puede basarse en una norma no publicada por un organismo normativo oficial, y por ello se ha tenido que realizar una adaptación de las WCAG al entorno español, con la aprobación de la norma UNE 139803:2004. Requisitos de Accesibilidad para Contenidos en la Web (BOE de 19-02-2005).

Como complemento a las políticas de información que promueven la accesibilidad se han realizado importantes campañas de sensibilización. En la Unión Europea se instituyó 2003 como Año europeo de las personas con discapacidad con múltiples eventos ${ }^{6}$ en diversos países. El 2004 fue también el Año iberoamericano de la discapacidad ${ }^{7}$. Finalmente cabe señalar que desde 2005, el 17 de mayo ha sido designado a nivel mundial com el Día de Internet, a fin de promover su uso.

\footnotetext{
3 http://register.consilium.eu.int/pdf/es/03/st05/st05165es03.pdf.

${ }^{4}$ http://eur-lex.europa.eu/LexUriServ/LexUriServ.do?uri=OJ:L:2004:134:0114:0240:ES:PDF.

5 http://eur-lex.europa.eu/LexUriServ/LexUriServ.do?uri=COM:2005:0425:FIN:ES:PDF.

${ }^{6}$ http://www.able.ie/eypdentmgmt.nsf/marchtomay?OpenForm.

${ }^{7}$ http://www.sidar.org/funacti/inter/2004ibero.php.
} 


\section{Metodología}

La metodología utilizada es prácticamente idéntica a la ya usada en nuestro estudio del año 2001 (Térmens, Ribera, Sulé, 2003). El universo estudiado han sido todas las universidades españolas sin ninguna distinción por titularidad privada o pública, tomando como base el listado oficial del Ministerio de Ciencia e Innovación ${ }^{8}$. Hay que tener en cuenta que las universidades privadas, como tales, no están sujetas a los compromisos de la administración pública en cuanto a cumplimiento de las pautas de accesibilidad y otros; aún así, en este estudio se les ha dado un tratamiento equivalente a las universidades públicas a fin de facilitar una visión global de la oferta en educación superior en España. Solamente se han excluido las universidades de verano y las internacionales, como la Menéndez Pelayo, por entender que no forman parte del sistema reglado español de enseñanza superior. Estos parámetros dan un total de 72 universidades, un número superior al de 65 universidades existentes el año 2001. Con el fin de comparar los resultados de las dos muestras, sólo se han observado los datos de las 65 universidades ya existentes el 2001.

De cada institución se han analizado dos páginas consideradas como representativas de cada web. Estas páginas son:

- Página inicial o homepage del web institucional de la universidad: por ser una página de paso obligado a cualquier otra y porque suele ser el máximo exponente de las tecnologías usadas en el web corporativo.

- Página del primer plan de estudios que se localiza por orden de lectura (normalmente por orden alfabético): por ser una página que suele estar generada de forma automática y permite observar la implementación de los criterios de diseño en plantillas y diseños automatizados.

La recogida de datos se realizó en el mes de abril de 2006. Los distintos indicadores fueron observados de forma manual con la ayuda, en algunos casos, de la herramienta de diagnóstico AIS Web Accessibility Toolbar?

En cada caso se comprobó el grado de cumplimiento de las recomendaciones WCAG 1.0 en su nivel A, de la norma 508 y de 5 indicadores adicionales.

La norma 508 solo es de uso obligatorio en Estados Unidos y su conocimiento en España solo está extendido entre especialistas en accesibilidad; por esta razón no se puede exigir su seguimiento. Si se ha incluido su valoración en este estudio es porque las normas específicas 508 marcan un grado superior de exigencia que aparecen recogidas en las nuevas normativas del Consorcio (WCAG 2.0) y para facilitar una posible comparación de los resultados con los obtenidos en estudios similares en Estados Unidos. Medir el uso de la norma 508 equivale a testear hasta qué punto la adaptación de los web a las reglas de accesibilidad se realiza simplemente por imperativo legal o además por convencimiento, yendo un punto más allá de la norma vigente.

\footnotetext{
${ }^{8}$ http://www.micinn.es/univ/jsp/plantilla.jsp?id=5000\&area=ccuniv.

9 http://www.visionaustralia.org.au/ais/toolbar/.
} 
Además de los indicadores WCAG y 508, se ha valorado el cumplimiento de cinco indicadores adicionales, que a consideración de los autores són importantes dentro de una visión no normativa de la accesibilidad: que el contenido multimedia sea accesible, disponibilidad de vínculos con texto significativo, disponibilidad de versión noframes, que las páginas dispongan de un título significativo y que se incorporen metadatos descriptivos del contenido.

\section{Resultados}

\subsection{Cumplimiento de la norma WCAG 1.0}

Los resultados por indicadores muestran grandes diferencias entre ellos. En las páginas principales (Tabla I), presentan un alto grado de cumplimiento los

\section{TABLA I}

\section{Datos globales WCAG página institucional}

\begin{tabular}{|c|c|c|c|c|c|c|c|}
\hline & \multirow{2}{*}{\multicolumn{3}{|c|}{2006}} & \multirow{2}{*}{\multicolumn{3}{|c|}{2001}} \\
\hline & & & & & & & \\
\hline \multicolumn{2}{|r|}{ Indicadores WCAG } & Casos & Acce- & $\begin{array}{c}\% \\
\text { Accesi- }\end{array}$ & Casos & Acce- & $\begin{array}{c}\% \\
\text { Accesi- }\end{array}$ \\
\hline 1.1 & Alternativa a gráficos & 64 & 34 & 53,13 & 62 & 10 & 16,13 \\
\hline $1.2,9.1$ & Mapas cliente o redundantes & 26 & 25 & 96,15 & 31 & 31 & 100,00 \\
\hline 1.3 & Descripción auditiva alternativa & 6 & 1 & 16,67 & 2 & 1 & 50,00 \\
\hline 1.4 & $\begin{array}{l}\text { Alternativa a presentación mul- } \\
\text { timedia }\end{array}$ & 2 & 0 & 0,00 & 0 & 0 & - \\
\hline 2.1 & Legible sin color & 61 & 55 & 90,16 & 65 & 65 & 100,00 \\
\hline 4.1 & Cambio explícito de idioma & 46 & 5 & 10,87 & 19 & 0 & 0,00 \\
\hline 5.1 & Tablas con cabecera $\mathrm{TH}$ & 2 & 0 & 0,00 & 1 & 0 & 0,00 \\
\hline 5.2 & Tablas con datos agrupados & 1 & 0 & 0,00 & 1 & 0 & 0,00 \\
\hline 6.1 & Legible sin CSS & 62 & 50 & 80,65 & 17 & 5 & 29,41 \\
\hline 6.2 & Alternativa a dinámico & 5 & 0 & 0,00 & 0 & 0 & - \\
\hline 6.3 & Legible sin scripts & 60 & 27 & 45,00 & 49 & 19 & 38,78 \\
\hline 7.1 & Sin parpadeo & 65 & 53 & 81,54 & 63 & 52 & 82,54 \\
\hline 8.1 & $\begin{array}{l}\text { Scripts legibles con tecnologías } \\
\text { asistencia }\end{array}$ & 0 & 0 & - & 0 & 0 & - \\
\hline 11.4 & Alternativa a Flash, PDF & 7 & 4 & 57,14 & 9 & 0 & 0,00 \\
\hline 12.1 & Marcos con título significativo & 10 & 3 & 30,00 & 15 & 8 & 53,33 \\
\hline 14.1 & Lenguaje claro & 65 & 59 & 90,77 & 65 & 64 & 98,46 \\
\hline
\end{tabular}


indicadores de "Mapas cliente o reedundantes", "Legible sin color", "Legible sin CSS", "Sin parpadeo" y "Lenguaje claro". En los planes de estudios (Tabla II), vuelven a destacar los mismos indicadores, excepto el último.

TABLA II

Datos globales WCAG página plan de estudios

\begin{tabular}{|c|c|c|c|c|c|c|c|}
\hline & \multirow{2}{*}{\multicolumn{3}{|c|}{2006}} & \multirow{2}{*}{\multicolumn{3}{|c|}{2001}} \\
\hline & & & & & & & \\
\hline \multicolumn{2}{|r|}{ Indicadores WCAG } & Casos & Acce- & $\begin{array}{c}\% \\
\text { Accesi- }\end{array}$ & Casos & Acce- & $\begin{array}{c}\% \\
\text { Accesi- }\end{array}$ \\
\hline 1.1 & Alternativa a gráficos & 49 & 15 & 30,61 & 55 & 9 & 16,36 \\
\hline $1.2,9.1$ & Mapas cliente o redundantes & 8 & 7 & 87,50 & 12 & 11 & 91,67 \\
\hline 1.3 & Descripción auditiva alternativa & 1 & 0 & 0,00 & 1 & 1 & 100,00 \\
\hline 1.4 & $\begin{array}{l}\text { Alternativa a presentación mul- } \\
\text { timedia }\end{array}$ & 2 & 1 & 50,00 & 0 & 0 & - \\
\hline 2.1 & Legible sin color & 62 & 59 & 95,16 & 65 & 65 & 100,00 \\
\hline 4.1 & Cambio explícito de idioma & 20 & 2 & 10,00 & 5 & 0 & 0,00 \\
\hline 5.1 & Tablas con cabecera TH & 58 & 8 & 13,79 & 45 & 0 & 0,00 \\
\hline 5.2 & Tablas con datos agrupados & 35 & 2 & 5,71 & 6 & 0 & 0,00 \\
\hline 6.1 & Legible sin CSS & 44 & 40 & 90,91 & 13 & 4 & 30,77 \\
\hline 6.2 & Alternativa a dinámico & 2 & 1 & 0,00 & 0 & 0 & - \\
\hline 6.3 & Legible sin scripts & 43 & 24 & 55,81 & 23 & 11 & 47,83 \\
\hline 7.1 & Sin parpadeo & 63 & 62 & 98,41 & 61 & 59 & 96,72 \\
\hline 8.1 & $\begin{array}{l}\text { Scripts legibles con tecnologías } \\
\text { asistencia }\end{array}$ & 0 & 0 & - & 0 & 0 & - \\
\hline 11.4 & Alternativa a Flash, PDF & 13 & 2 & 15,38 & 7 & 0 & 0,00 \\
\hline 12.1 & Marcos con título significativo & 14 & 5 & 35,71 & 20 & 10 & 50,00 \\
\hline 14.1 & Lenguaje claro & 63 & 33 & 52,38 & 65 & 64 & 98,46 \\
\hline
\end{tabular}

Si comparamos estos resultados con los obtenidos el año 2001, apreciamos mejoras especialmente en la "Alternativa a gráficos", "Legible sin CSS" y "Alternativa a Flash, PDF". Cabe resaltar que estas mejoras se han conseguido al mismo tiempo que ha aumentado de forma destacada el uso de estas técnicas, como lo prueba el aumento de casos contabilizados el año 2006 respecto el 2001 en la mayoría de indicadores. Tras realizar una prueba de independencia chi cuadrado no se ha podido establecer ninguna relación significativa entre los resultados del año 2006 respecto a los obtenidos el 2001; este resultado es extensible a los datos parciales por universidad descritos en el Anexo I. 
A nivel global, se observa que 10 universidades (Autónoma de Madrid, Complutense de Madrid, Castilla-La Mancha, Deusto, Oviedo, Sevilla, Nacional a Distancia, Oberta de Catalunya, Politécnica de Cartagena, Politécnica de Madrid) cumplen al $100 \%$ con los indicadores WCAG en su página principal, en cambio solo una (Sevilla) los cumple en su página de plan de estudios. El resto de universidades presentan niveles de cumplimiento inferiores que pueden llegar a un mínimo del $20 \%$ en la página principal de Politécnica de Catalunya y del 33,33\% en diversas páginas de planes de estudios (Antonio de Nebrija, Autónoma de Barcelona, Illes Balears, Navarra, Miguel Hernández, Pablo de Olavide, Pública de Navarra) (véase Anexo 1). Respecto a las mediciones de 2001, en las páginas principales se constata un aumento de las universidades que alcanzan la excelencia cumpliendo al 100\% los indicadores, pero el conjunto de webs no presentan un cambio significativo; en cambio, en las páginas de planes de estudios, los resultados tienden a ser claramente peores (véanse las Figuras 1 y 2).

FIGURA 1

Cumplimiento de las directrices WCAG en las páginas institucionales, 2001-2006

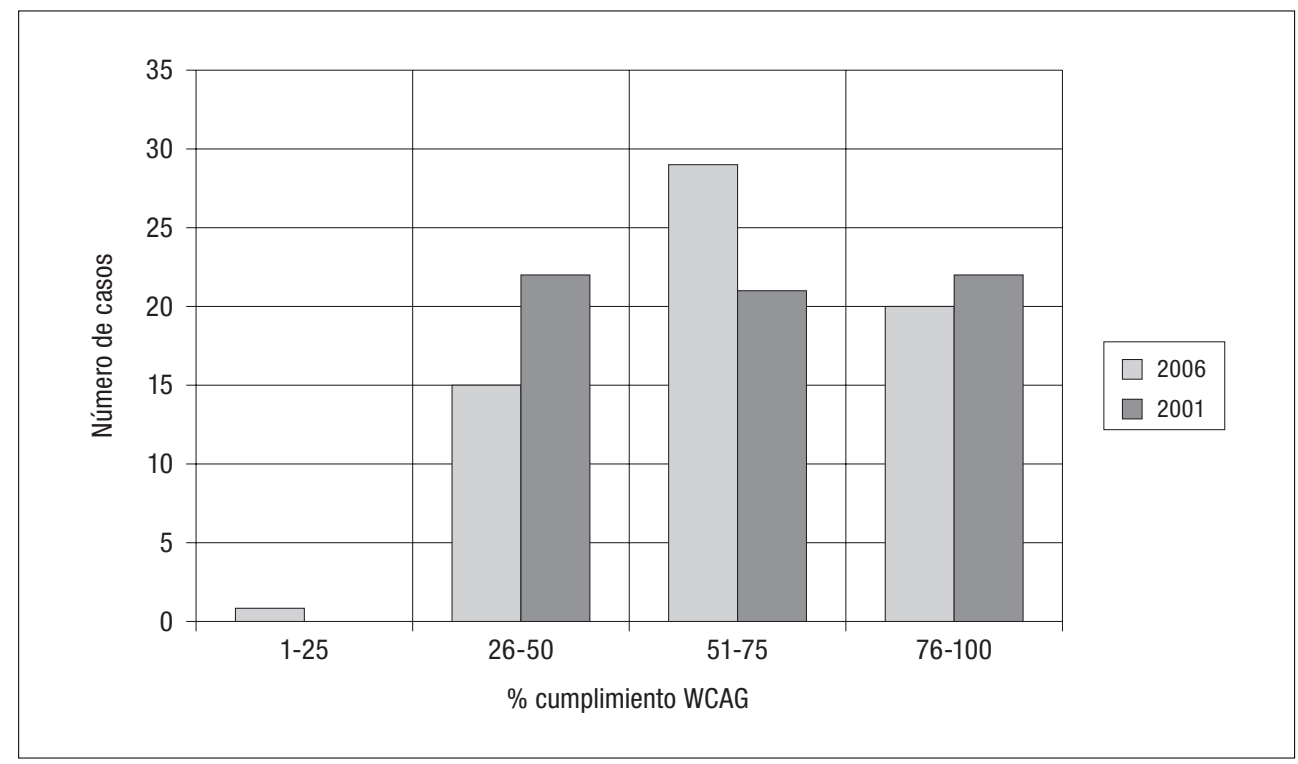




\section{GRÁFICO 2}

Cumplimiento de las directrices WCAG en las páginas de planes de estudios, 2001-2006

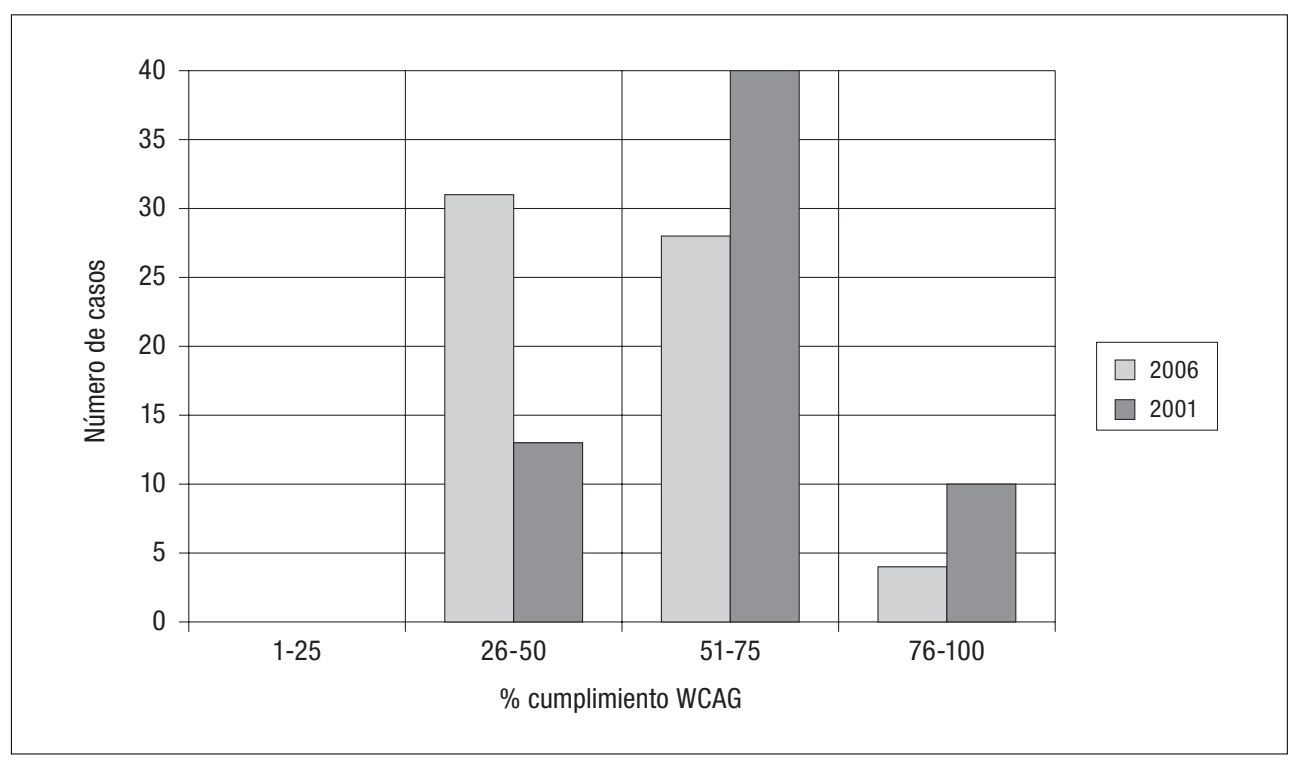

\subsection{Cumplimiento de la norma 508}

Se ha comprobado que el cumplimiento de la norma 508 presenta unos niveles más bajos que el de las normas WCAG. Recordemos que las dos normas comparten un corpus común de parámetros, pero difieren también en parámetros propios de cada una. Si nos fijamos en los indicadores presentes en la norma 508 y no en las WCAG, comprobaremos que estos presentan un bajo nivel de seguimiento entre las universidades españolas (Tablas III y IV). Destacamos los valores

\section{TABLA III}

Datos globales 508 página institucional

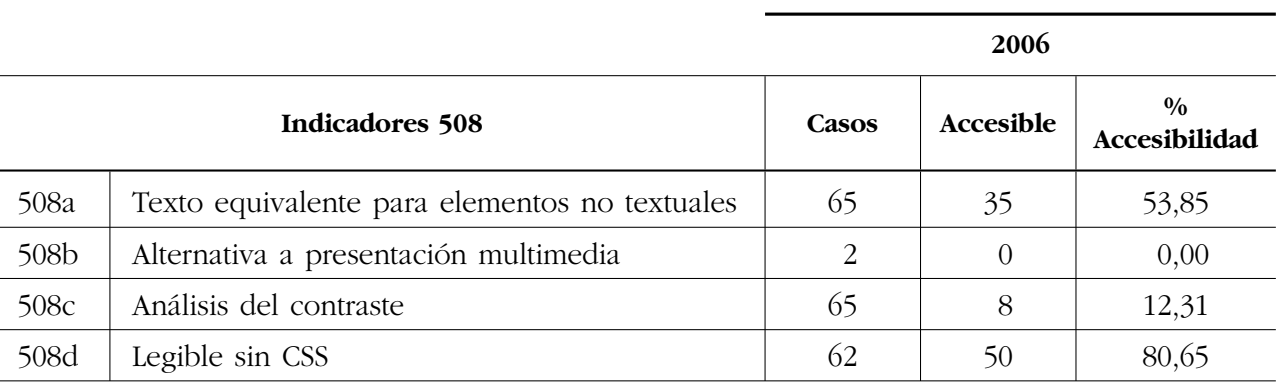




\section{TABLA III (continuación)}

\begin{tabular}{|c|c|c|c|c|}
\hline & & & \\
\hline & & \multicolumn{3}{|c|}{2006} \\
\hline \multicolumn{2}{|r|}{ Indicadores 508} & Casos & Accesible & $\%$ \\
\hline $508 \mathrm{e}$ & Mapas cliente o redundantes & 3 & 2 & 66,67 \\
\hline $508 f$ & Mapas cliente (zonas geométricas) & 5 & 4 & 80,00 \\
\hline $508 \mathrm{~g}$ & Tablas con cabecera TH & 2 & 0 & 0,00 \\
\hline $508 \mathrm{~h}$ & Tablas con datos agrupados & 1 & 0 & 0,00 \\
\hline $508 \mathrm{i}$ & Marcos con título significativo & 10 & 3 & 30,00 \\
\hline $508 \mathrm{j}$ & Sin parpadeo & 52 & 40 & 76,92 \\
\hline $508 \mathrm{k}$ & Versión texto accesible & 7 & 3 & 42,86 \\
\hline 5081 & Scripts legibles con tecnologías asistencia & 0 & 0 & - \\
\hline $508 \mathrm{~m}$ & Scripts alternativos accesibles & 34 & 2 & 5,88 \\
\hline $508 n$ & Etiquetas en formularios & 43 & 13 & 30,23 \\
\hline 5080 & Saltar al contenido & 61 & 10 & 16,39 \\
\hline $508 \mathrm{p}$ & Redireccionamientos automáticos & 3 & 0 & 0,00 \\
\hline
\end{tabular}

TABLA IV

Datos globales 508 página plan de estudios

\begin{tabular}{|c|c|c|c|c|}
\hline & \multirow{2}{*}{\multicolumn{3}{|c|}{2006}} \\
\hline \multirow{2}{*}{\multicolumn{2}{|c|}{ Indicadores 508}} & & & \\
\hline & & \multirow{2}{*}{$\begin{array}{c}\text { Casos } \\
49 \\
\end{array}$} & \multirow{2}{*}{$\begin{array}{c}\text { Accesible } \\
15 \\
\end{array}$} & \multirow{2}{*}{$\begin{array}{c}\mathbf{\%} \\
\text { Accesibilidad } \\
30,61\end{array}$} \\
\hline $508 \mathrm{a}$ & Texto equivalente para elementos no textuales & & & \\
\hline $508 \mathrm{~b}$ & Alternativa a presentación multimedia & 2 & 1 & - \\
\hline $508 \mathrm{c}$ & Análisis del contraste & 63 & 13 & 20,63 \\
\hline $508 \mathrm{~d}$ & Legible sin CSS & 44 & 39 & 88,64 \\
\hline $508 \mathrm{e}$ & Mapas cliente o redundantes & 2 & 1 & 50,00 \\
\hline $508 \mathrm{f}$ & Mapas cliente (zonas geométricas) & 7 & 6 & 85,71 \\
\hline $508 \mathrm{~g}$ & Tablas con cabecera TH & 58 & 8 & 13,79 \\
\hline $508 \mathrm{~h}$ & Tablas con datos agrupados & 35 & 2 & 5,71 \\
\hline $508 \mathrm{i}$ & Marcos con título significativo & 14 & 5 & 35,71 \\
\hline $508 \mathrm{j}$ & Sin parpadeo & 63 & 62 & 98,41 \\
\hline $508 \mathrm{k}$ & Versión texto accesible & 2 & 1 & 50,00 \\
\hline 5081 & Scripts legibles con tecnologías asistencia & 0 & 0 & - \\
\hline
\end{tabular}




\section{TABLA IV (continuación)}

\begin{tabular}{|c|c|c|c|c|}
\hline & & & \\
\hline & & \multicolumn{3}{|c|}{2006} \\
\hline \multicolumn{2}{|r|}{ Indicadores 508} & Casos & Accesible & $\begin{array}{c}\% \\
\end{array}$ \\
\hline $508 \mathrm{~m}$ & Scripts alternativos accesibles & 5 & 2 & 40,00 \\
\hline $508 n$ & Etiquetas en formularios & 21 & 8 & 38,10 \\
\hline 5080 & Saltar al contenido & 48 & 7 & 14,58 \\
\hline $508 \mathrm{p}$ & Redireccionamientos automáticos & 0 & 0 & - \\
\hline
\end{tabular}

deficientes obtenidos en los indicadores "508c. Análisis del contraste”, "508n. Etiquetas en formularios" y "5080. Saltar al contenido". Hemos observado que no existe una dependencia entre los resultados obtenidos en las páginas institucionales respecto a las páginas planes de estudios según el test de chi cuadrado.

La mayor rigurosidad de la norma 508 se constata en el hecho de que ninguna universidad española, en ninguno de los dos tipos de páginas analizados, alcanza a cumplir al 100\% con la misma (Anexo II y Figura 3).

FIGURA 3

Cumplimiento de la norma 508 en las páginas institucionales $y$ de planes de estudios, 2006

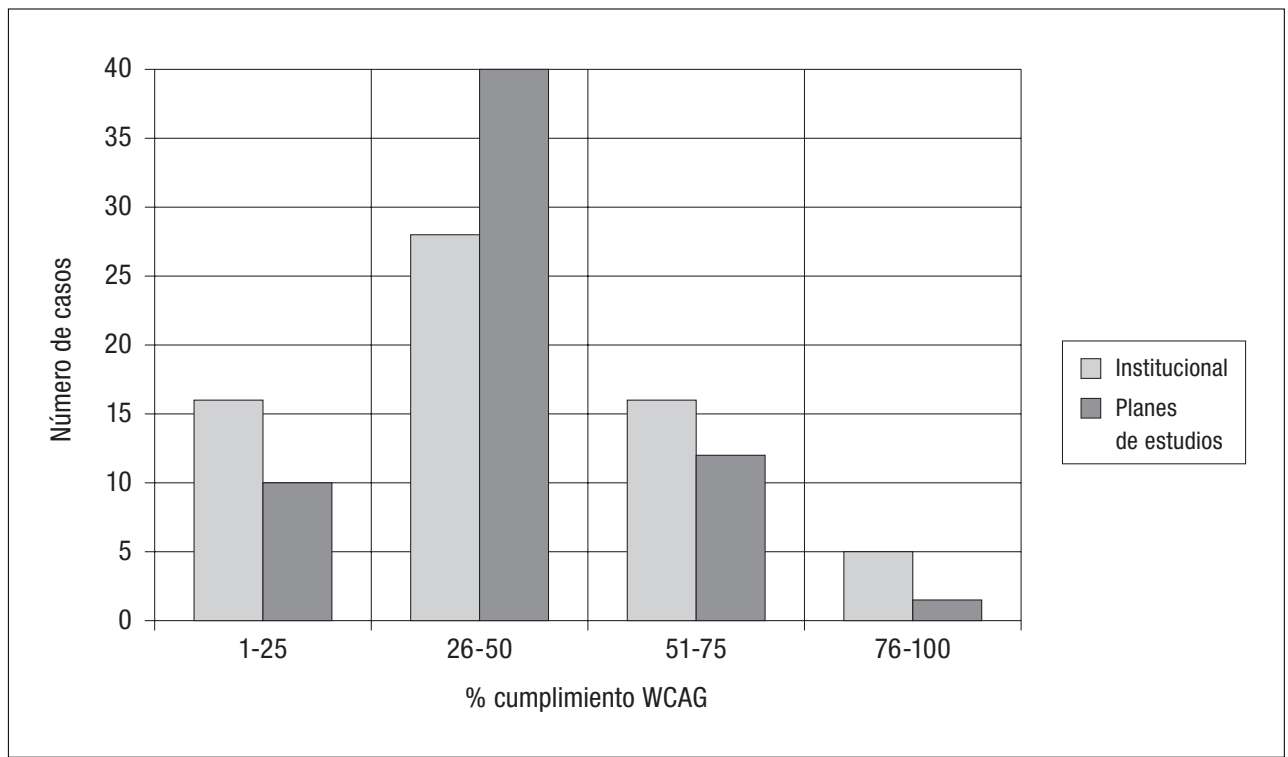




\section{FIGURA 4}

Cumplimiento de otros indicadores en las páginas institucionales, 2001-2006

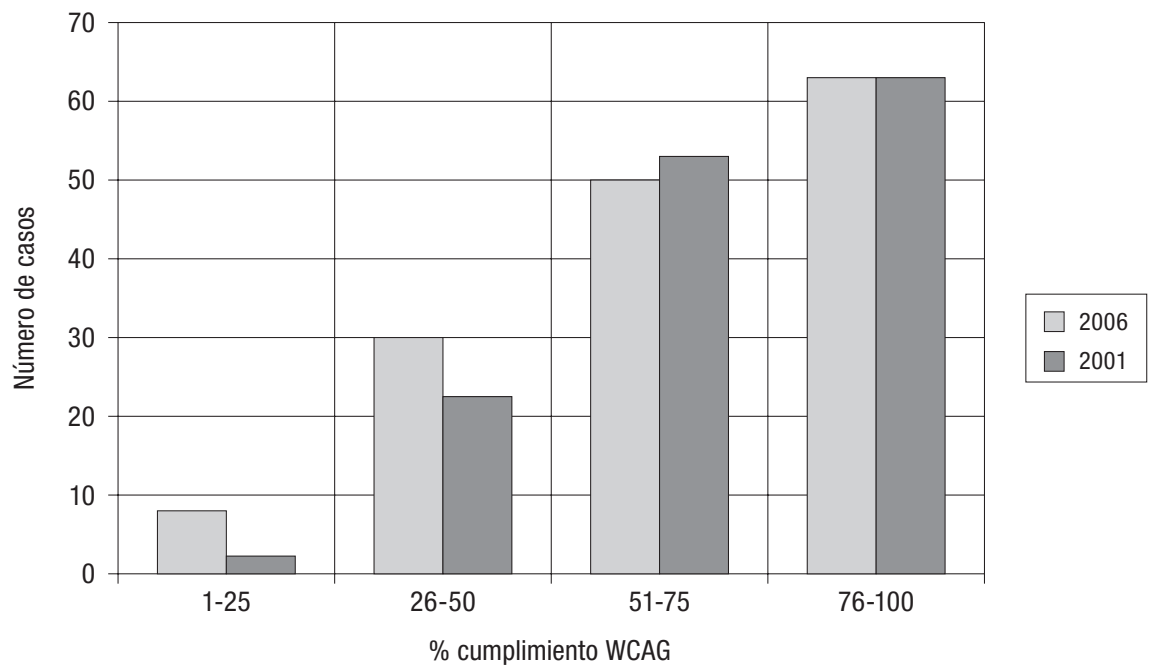

FIGURA 5

Cumplimiento de otros indicadores en las páginas de planes de estudios, 2001-2006

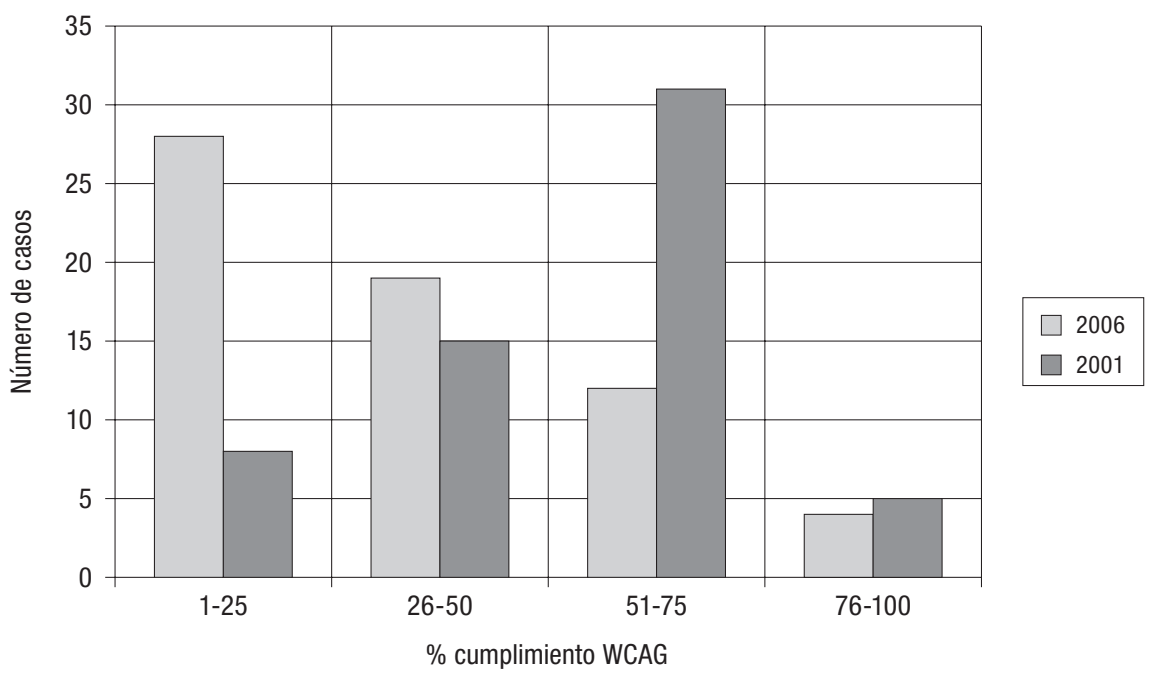




\subsection{Cumplimiento de otros indicadores}

Respecto a los cinco indicadores adicionales a las WCAG y a la norma 508, los resultados obtenidos el 2006 son parejos a los del año 2001, con un cumplimiento global del 65\% en el caso de las páginas institucionales y del 62\% en el de las páginas de planes de estudios (Anexo III y Figuras 4 y 5).

\section{Discusión}

El objetivo de este estudio era medir sobre el terreno los efectos de la nueva legislación española en accesibilidad y de las campañas de sensibilización en el período 2001-2006. En base a los resultados obtenidos, se puede afirmar que estos efectos han sido bastante menores de lo que cabría esperar y es razonable valorar las mejoras en accesibilidad como decepcionantes.

Estos resultados globales no son muy diferentes de los obtenidos en Australia (Alexander, 2003; Alexander, 2007), pero sí difieren de los obtenidos en estudios similares del Reino Unido (Kelly, 2005) y de la Universidad de Wisconsin, USA (Schmetzke, 2005), en los que se aprecia una mejoría con el paso del tiempo. Diversos factores pueden explicar estas diferencias entre países: el grado de implicación de los webmasters con la accesibilidad, los cambios en las tecnologías introducidas en los webs, y la fuerza con la que se hace cumplir la legislación. En cualquier caso, tal y cómo indican algunos autores (Witt, McDermott, 2002) la puesta en práctica de las directrices WCAG no es una tarea simple.

Esto no significa que la construcción de las páginas web no haya cambiado. Como aspecto positivo, una observación atenta muestra que la mayoría de las páginas estudiadas han sido rediseñadas respecto a las existentes el año 2001 y que los cambios aplicados lo han sido en la línea de las recomendaciones de los estándares del W3C. Se nota, por ejemplo, un menor uso de las tablas como herramienta de maquetación y, sobretodo, una aplicación masiva de las hojas de estilo CSS para marcar los elementos tipográficos y, en menor medida, para la maquetación. En sentido negativo cabe remarcar el aumento de los errores en los textos incorporados: errores de sintaxis, de ortografía y de marcaje de los cambios de idioma.

Los resultados obtenidos en las páginas principales y en las de los planes de estudios son diferentes y, en general, son mejores en el primer grupo. Estos dos tipos de páginas presentan contenidos bien diferentes y también se han construido de distinta forma. Las páginas principales en la mayoría de los casos se han diseñado y maquetado de forma manual con el fin de conseguir el mejor impacto gráfico; con este objetivo también suelen incorporar soluciones técnicas más sofisticadas.

Por su parte, las páginas de planes de estudios son generadas de forma dinámica en su totalidad, gracias a sistemas de programación que extraen sus contenidos de las bases de datos corporativas. A nivel técnico son páginas más austeras y con un uso generalizado de las tablas de datos. Es triste comprobar 
que las páginas generadas de forma automática no sean accesibles, cuando las herramientas de programación pueden hacer esta tarea bien fácil. Al contrario, se ha constatado que estas páginas son en general menos accesibles que las del otro grupo; ello nos induce a pensar que el problema de la falta de accesibilidad no se encuentra en el lado de las herramientas sino en el de las personas que las usan, en este caso el personal informático.

Finalmente cabe también preguntarse sobre la coherencia de la legislación aprobada en España. En Estados Unidos la promulgación de la norma 508 el año 1998 no solo llevó aparejado su obligado cumplimiento (incluyendo sanciones para los infractores), sino también el establecimiento de mecanismos de difusión de su contenido, de las soluciones a adoptar y de procedimientos para demostrar su observancia. Entre los años 2001 y 2006 en España y también a nivel de la Unión Europea se promulgaron diversos rangos de normas con el fin de impulsar la accesibilidad pero que han adolecido de algunos fallos en su promulgación: en algunos casos no se indicaba claramente qué normas técnicas de accesibilidad había que cumplir, los plazos límite para adaptar las web existentes se han prorrogado más de una vez, no se han establecido los mecanismos para certificar el cumplimiento de la accesibilidad, no se ha concretado un régimen sancionador para los infractores, etc. Es cierto que toda la regulación promulgada el año 2007 está corrigiendo estas deficiencias y en el futuro cabrá esperar una mayor eficacia en la legislación en accesibilidad electrónica, pero en el año 2006 las webs que hemos estudiado aún no habían cambiado radicalmente su nivel de accesibilidad respecto al año 2001, mermando a numerosos ciudadanos con discapacidades su derecho a navegar sin barreras.

En los años transcurridos entre los dos estudios, el mismo concepto de accesibilidad también ha cambiado, como también lo han hecho las herramientas que utilizamos para la consulta de las páginas web. Las mejores prácticas actuales sobre accesibilidad han ido incorporando la filosofía del diseño centrado en el usuario, garante de una accesibilidad real, y que propugna incluir a usuarios con discapacidades en las distintas fases de desarrollo de los sitios web (Petrie, Kheir 2007) (Hassan, Martín, 2004). También las propias directrices WCAG se están reformulando, con la propuesta de nueva versión 2.0 a punto de ver la luz en el momento de redactar este artículo (Web, 2008) Este contexto nos lleva a pensar que la legislación española en accesibilidad digital aún deberá avanzar más, especialmente en medidas de impulso, promoción e incluso sanción y que los responsables de las webs universitarias deberán realizar un esfuerzo suplementario para alcanzar estos nuevos niveles de calidad.

\section{Bibliografía}

La accesibilidad de los portales universitarios en España (2004): Madrid, Fundosa Teleservicios. 41 p. Disponible en: http://www.discapnet.es/documentos/infoaccesibilidad/ tema 01/pdf/Portales universitarios detallada.pdf [Consulta: 12-1-2009]. 
Breve análisis sobre el estado de accesibilidad de los portales de las universidades públicas españolas (2004): Acctiva. 10 p. Disponible en: http://www.acctiva.com/recursos/ AcctivaUniversidades.pdf [Consulta: 12-1-2009].

Alexander, D. (2003): "How accessible are Australian university web sites". Ariadne. 38. Disponible en: http://www.ariadne.ac.uk/issue38/web-watch/ [Consulta: 12-1-2009].

Alexander, D., y Rippon, S. (2007). "University website accessibility revisited" Ausweb 2007. Disponible en: http://ausweb.scu.edu.au/aw07/papers/refereed/alexander/paper.html [Consulta: 12-1-2009].

Dissability Rights Comission (2004): The Web. Access and Inclusion for Disabled People. A formal investigation. London, The Stationery Office.

García Gómez, J. G. (2006): “Principales barreras de acceso al contenido en internet: análisis de malas prácticas en la representación de información web". XI Encuentros Internacionales sobre Sistemas de Información y Documentación Ibersid 2006. Zaragoza, Universidad de Zaragoza.

Hassan Montero, Y., y Martín Fernández, F. J. (2004): “Propuesta de adaptación de la metología de diseño centrado en el usuario para el desarrollo de sitios web accesibles". Revista Española de Documentación Científica. 27 (3), pp. 330-344.

Kelly, B.; Sloan, D.; Phipps, L.; Petrie, H., y Hamilton, F. (2005): "Forcing standardization or accommodating diversity? A framework for applying the WCAG in the Real World. International Cross-Disciplinary Workshop on Web Accessibility (W4A)". ACM International Conference Proceeding Series, vol. 88, New York, ACM. pp. 46-54.

Mankoff, J.; Fait, H., y Tran, T. (2005): "Is your web page accessible?: A comparative study of methods for assessing web page accessibility for the blind". Proceedings of the SIGCHI conference on Human factors in computing systems. New York, ACM Press. pp. 41-50.

Milne, S.; Dickinson A.; Carmichael A.; Sloan D.; Eisma R., y Gregor P. (2005): “Are guidelines enough? An Introduction to designing web sites accessible to older people». IBM systems journal, 44 (3), 557-571.

Petrie, H., y Kheir, O. (2007): "The relationship between accessibility and usability of websites". Proceedings of the CHI conference on Empirical studies in web interaction. San Jose, ACM Press, pp. 397-406.

Pinto, M.; Berrocal, J. L. A.; García, J. A. C., Marcial, V. F.; Figuerola, C. G.; Marco, J. G.; Camarero, C. G., y Rodríguez, A. F. Z. (2005): "Quality assessment of Spanish universities' web sites focused on the European research area". Scientometrics, 65 (1), pp. 67-93.

Schmetzke, Axel (2005): Web page accessibility on University of Wisconsin campuses: 2005 survey data and seven-year trend data. Disponible en: http://library.uwsp.edu/aschmetz/Accessible/UW-Campuses/Survey2005/contents2005.htm [Consulta: 12-1-2009].

Sloan, D.; Dickinson, A.; Mcilroy, N., y Gibson, L. (2006): Evaluating the usability of online accessibility information. University of Dundee.

Térmens Graells, M; Ribera Turró, M., y Sulé Duesa, A. (2003): "Nivel de accesibilidad de las sedes web de las universidades españolas" en Revista Española de Documentación Científica, 26 (1), pp. 19-37.

Térmens Graells, M.; Ribera Turró, M., y Sulé Duesa, A. (2002): “L'accessibilitat dels webs de les universitats catalanes", Item, 31, pp. 53-76. 
Térmens Graells, M. (2002): "La accesibilidad de las webs de las universidades andaluzas" en XII Jornadas Bibliotecarias de Andalucía. Málaga, Asociación Andaluza de Bibliotecarios, pp. 213-225.

Web Content Accesibility Guidelines 1.0. W3C Recommendation 5 May 1999. Disponible en: http://www.w3.org/TR/WAI-WEBCONTENT [Consulta: 12-1-2009].

Web Content Accesibility Guidelines 2.0. W3C Candidate Recommendation 30 April 2008. Disponible en: http://www.w3.org/TR/WCAG20/ [Consulta: 12-1-2009].

Witt, N. A. J., y McDermott, A. P. (2002): "Achieving SENDA-compliance for web sites in further and higher education: an art or a science?» en Phipps, L., Surherland, A., Seale, J. Access all areas: disability, technology and learning. Oxforf: ALT/TechDis. pp. $42-49$.

\section{Anexos}

\section{ANEXO I}

Indicadores WCAG por universidades

\begin{tabular}{|c|c|c|c|c|}
\hline & \multirow{2}{*}{\multicolumn{2}{|c|}{ Páginas institucionales }} & & \\
\hline & & & \multicolumn{2}{|c|}{ Páginas planes de estudios } \\
\hline Universidad & $\begin{array}{c}\% \\
\text { cumplimiento } \\
\text { indicadores } \\
\text { WCAG } 2006\end{array}$ & $\begin{array}{c}\% \\
\text { cumplimiento } \\
\text { indicadores } \\
\text { WCAG } 2001\end{array}$ & $\begin{array}{c}\% \\
\text { cumplimiento } \\
\text { indicadores } \\
\text { WCAG } 2006\end{array}$ & $\begin{array}{c}\% \\
\text { cumplimiento } \\
\text { indicadores } \\
\text { WCAG } 2001\end{array}$ \\
\hline Alfonso X el Sabio & 40,00 & 80,00 & 40,00 & 42,86 \\
\hline Antonio de Nebrija & 50,00 & 57,14 & 33,33 & 66,67 \\
\hline Autónoma de Barcelona & 71,43 & 71,43 & 33,33 & 60,00 \\
\hline Autónoma de Madrid & 100,00 & 83,33 & 40,00 & 80,00 \\
\hline Camilo José Cela & 71,43 & 50,00 & 50,00 & 62,50 \\
\hline Cardenal Herrera - CEU & 66,67 & 60,00 & 60,00 & 75,00 \\
\hline Carlos III de Madrid & 66,67 & 71,43 & 62,50 & 83,33 \\
\hline Católica de Avila & 37,50 & 80,00 & 50,00 & 66,67 \\
\hline Católica San Antonio & 33,33 & 50,00 & 60,00 & 60,00 \\
\hline Complutense de Madrid & 100,00 & 50,00 & 75,00 & 50,00 \\
\hline A Coruña & 33,33 & 71,43 & 62,50 & 75,00 \\
\hline Alcalá & 28,57 & 42,86 & 40,00 & 50,00 \\
\hline Alicante & 87,50 & 71,43 & 44,44 & 60,00 \\
\hline Almería & 80,00 & 66,67 & 55,56 & 60,00 \\
\hline Barcelona & 57,14 & 50,00 & 50,00 & 100,00 \\
\hline Burgos & 62,50 & 80,00 & 75,00 & 66,67 \\
\hline Cádiz & 62,50 & 80,00 & 40,00 & 83,33 \\
\hline
\end{tabular}




\section{ANEXO I (continuación)}

\begin{tabular}{|c|c|c|c|c|}
\hline \multirow[b]{2}{*}{ Universidad } & \multicolumn{2}{|c|}{ Páginas institucionales } & \multicolumn{2}{|c|}{ Páginas planes de estudios } \\
\hline & $\begin{array}{c}\% \\
\text { cumplimiento } \\
\text { indicadores } \\
\text { WCAG } 2006\end{array}$ & $\begin{array}{c}\% \\
\text { cumplimiento } \\
\text { indicadores } \\
\text { WCAG } 2001\end{array}$ & $\begin{array}{c}\% \\
\text { cumplimiento } \\
\text { indicadores } \\
\text { WCAG } 2006\end{array}$ & $\begin{array}{c}\% \\
\text { cumplimiento } \\
\text { indicadores } \\
\text { WCAG 2001 }\end{array}$ \\
\hline Cantabria & 75,00 & 66,67 & 50,00 & 60,00 \\
\hline Castilla-La Mancha & 100,00 & 50,00 & 71,43 & 71,43 \\
\hline Córdoba & 50,00 & 33,33 & 75,00 & 60,00 \\
\hline Deusto & 100,00 & 37,50 & 88,89 & 50,00 \\
\hline Extremadura & 57,14 & 83,33 & 50,00 & 85,71 \\
\hline Girona & 50,00 & 42,86 & 83,33 & 50,00 \\
\hline Granada & 75,00 & 37,50 & 75,00 & 75,00 \\
\hline Huelva & 42,86 & 83,33 & 60,00 & 60,00 \\
\hline Illes Balears & 66,67 & 80,00 & 33,33 & 57,14 \\
\hline Jaén & 66,67 & 83,33 & 45,45 & 75,00 \\
\hline La Laguna & 28,57 & 50,00 & 40,00 & 75,00 \\
\hline La Rioja & 62,50 & 80,00 & 75,00 & 80,00 \\
\hline Las Palmas de Gran Canaria & 50,00 & 71,43 & 60,00 & 75,00 \\
\hline León & 66,67 & 37,50 & 50,00 & 80,00 \\
\hline Lleida & 71,43 & 71,43 & 50,00 & 57,14 \\
\hline Málaga & 66,67 & 50,00 & - & 60,00 \\
\hline Mondragón & 71,43 & 75,00 & 87,50 & 66,67 \\
\hline Murcia & 83,33 & 66,67 & 71,43 & 75,00 \\
\hline Navarra & 85,71 & 83,33 & 33,33 & 75,00 \\
\hline Oviedo & 100,00 & 66,67 & 50,00 & 100,00 \\
\hline Salamanca & 57,14 & 83,33 & 60,00 & 60,00 \\
\hline Santiago de Compostela & 87,50 & 40,00 & 55,56 & 66,67 \\
\hline Sevilla & 100,00 & 75,00 & 100,00 & 80,00 \\
\hline València & 85,71 & 66,67 & 50,00 & 60,00 \\
\hline Valladolid & 87,50 & 80,00 & 55,56 & 75,00 \\
\hline Vic & 66,67 & 80,00 & 50,00 & 60,00 \\
\hline Vigo & 44,44 & 75,00 & 44,44 & 50,00 \\
\hline Zaragoza & 37,50 & 80,00 & 57,14 & 57,14 \\
\hline País Vasco & 80,00 & 62,50 & 50,00 & 33,33 \\
\hline Europea de Madrid & 80,00 & 50,00 & 66,67 & 75,00 \\
\hline
\end{tabular}




\section{ANEXO I (continuación)}

\begin{tabular}{l|c|c|c|c}
\cline { 2 - 5 } \multicolumn{1}{c|}{ Universidad } & $\begin{array}{c}\text { Páginas institucionales } \\
\text { cumplimiento } \\
\text { indicadores } \\
\text { WCAG 2006 }\end{array}$ & $\begin{array}{c}\text { \% } \\
\text { cumplimiento } \\
\text { indicadores } \\
\text { WCAG 2001 }\end{array}$ & $\begin{array}{c}\text { \% } \\
\text { cumplimiento } \\
\text { indicadores } \\
\text { WCAG 2006 }\end{array}$ & $\begin{array}{c}\text { \% } \\
\text { cumplimiento } \\
\text { indicadores } \\
\text { WCAG 2001 }\end{array}$ \\
\hline Internacional de Catalunya & 85,71 & 50,00 & 37,50 & 50,00 \\
\hline Jaume I de Castellón & 62,50 & 80,00 & 60,00 & 66,67 \\
\hline Miguel Hernández de Elche & 70,00 & 83,33 & 33,33 & 33,33 \\
\hline Nacional de Educación a Dis- & & & & \\
tancia & 100,00 & 60,00 & 57,14 & 83,33 \\
\hline Oberta de Catalunya & 100,00 & 42,86 & 75,00 & 42,86 \\
\hline Pablo de Olavide & 57,14 & 100,00 & 33,33 & 50,00 \\
\hline Politécnica de Cartagena & 100,00 & 80,00 & 50,00 & 57,14 \\
\hline Politécnica de Cataluña & 20,00 & 71,43 & 57,14 & 57,14 \\
\hline Politécnica de Madrid & 100,00 & 50,00 & 50,00 & 66,67 \\
\hline Politécnica de Valencia & 71,43 & 50,00 & 40,00 & 37,50 \\
\hline Pompeu Fabra & 57,14 & 50,00 & 66,67 & 57,14 \\
\hline Pontificia Comillas & 62,50 & 71,43 & 66,67 & 57,14 \\
\hline Pontificia de Salamanca & 33,33 & 40,00 & 55,56 & 75,00 \\
\hline Pública de Navarra & 62,50 & 83,33 & 33,33 & 66,67 \\
\hline Ramón Llull & 62,50 & 83,33 & 55,56 & 57,14 \\
\hline Rey Juan Carlos & 66,67 & 62,50 & 50,00 & 62,50 \\
\hline Rovira i Virgili & 37,50 & 80,00 & 57,14 & 50,00 \\
\hline San Pablo - CEU & 57,14 & 42,86 & - & 33,33 \\
\hline
\end{tabular}

ANEXO II

Indicadores 508 por universidades

\begin{tabular}{l|c|c}
\cline { 2 - 3 } \multicolumn{1}{c|}{ Universidad } & Páginas institucionales & Páginas planes de estudios \\
\hline Alfonso X el Sabio & $\begin{array}{c}\text { \% cumplimiento } \\
\text { indicadores WCAG 2006 }\end{array}$ & $\begin{array}{c}\text { \% cumplimiento } \\
\text { indicadores WCAG 2006 }\end{array}$ \\
\hline Antonio de Nebrija & 37,50 & 44,44 \\
\hline Autónoma de Barcelona & 16,67 & 14,29 \\
\hline Autónoma de Madrid & 42,86 & 25,00 \\
\hline
\end{tabular}


ANEXO II (continuación)

\begin{tabular}{|c|c|c|}
\hline & \multirow{2}{*}{ Páginas institucionales } & \multirow{3}{*}{$\begin{array}{c}\text { Páginas planes de estudios } \\
\text { \% cumplimiento } \\
\text { indicadores WCAG } 2006\end{array}$} \\
\hline & & \\
\hline Universidad & $\begin{array}{c}\text { \% cumplimiento } \\
\text { indicadores WCAG } 2006\end{array}$ & \\
\hline Camilo José Cela & 20,00 & 28,57 \\
\hline Cardenal Herrera - CEU & 50,00 & 33,33 \\
\hline Carlos III de Madrid & 37,50 & 37,50 \\
\hline Católica de Avila & 14,29 & 33,33 \\
\hline Católica San Antonio & 0,00 & 66,67 \\
\hline Complutense de Madrid & 71,43 & 66,67 \\
\hline A Coruña & 37,50 & 42,86 \\
\hline Alcalá & 14,29 & 66,67 \\
\hline Alicante & 60,00 & 44,44 \\
\hline Almería & 40,00 & 57,14 \\
\hline Barcelona & 16,67 & 28,57 \\
\hline Burgos & 40,00 & 50,00 \\
\hline Cádiz & 40,00 & 66,67 \\
\hline Cantabria & 50,00 & 28,57 \\
\hline Castilla-La Mancha & 80,00 & 50,00 \\
\hline Córdoba & 16,67 & 50,00 \\
\hline Deusto & 66,67 & 62,50 \\
\hline Extremadura & 66,67 & 57,14 \\
\hline Girona & 14,29 & 50,00 \\
\hline Granada & 40,00 & 33,33 \\
\hline Huelva & 33,33 & 33,33 \\
\hline Illes Balears & 60,00 & 33,33 \\
\hline Jaén & 28,57 & 37,50 \\
\hline La Laguna & 0,00 & 66,67 \\
\hline La Rioja & 33,33 & 37,50 \\
\hline Las Palmas de Gran Canaria & 42,86 & 66,67 \\
\hline León & 16,67 & 25,00 \\
\hline Lleida & 66,67 & 66,67 \\
\hline Málaga & 37,50 & - \\
\hline Mondragón & 50,00 & 85,71 \\
\hline Murcia & 66,67 & 37,50 \\
\hline
\end{tabular}




\section{ANEXO II (continuación)}

\begin{tabular}{|c|c|c|}
\hline & \multirow{2}{*}{ Páginas institucionales } & \multirow{3}{*}{$\begin{array}{c}\text { Páginas planes de estudios } \\
\% \text { cumplimiento } \\
\text { indicadores WCAG } 2006\end{array}$} \\
\hline & & \\
\hline Universidad & $\begin{array}{c}\text { \% cumplimiento } \\
\text { indicadores WCAG } 2006\end{array}$ & \\
\hline Navarra & 60,00 & 22,22 \\
\hline Oviedo & 83,33 & 42,86 \\
\hline Salamanca & 33,33 & 33,33 \\
\hline Santiago de Compostela & 66,67 & 33,33 \\
\hline Sevilla & 83,33 & 71,43 \\
\hline València & 75,00 & 16,67 \\
\hline Valladolid & 83,33 & 50,00 \\
\hline Vic & 37,50 & 33,33 \\
\hline Vigo & 0,00 & 25,00 \\
\hline Zaragoza & 16,67 & 33,33 \\
\hline País Vasco & 66,67 & 33,33 \\
\hline Europea de Madrid & 71,43 & 50,00 \\
\hline Internacional de Catalunya & 60,00 & 50,00 \\
\hline Jaume I de Castellón & 28,57 & 25,00 \\
\hline Miguel Hernández de Elche & 44,44 & 25,00 \\
\hline Nacional de Educación a Distancia & 37,50 & 50,00 \\
\hline Oberta de Catalunya & 75,00 & 42,86 \\
\hline Pablo de Olavide & 33,33 & 33,33 \\
\hline Politécnica de Cartagena & 50,00 & 28,57 \\
\hline Politécnica de Cataluña & 0,00 & 40,00 \\
\hline Politécnica de Madrid & 50,00 & 25,00 \\
\hline Politécnica de Valencia & 80,00 & 37,50 \\
\hline Pompeu Fabra & 33,33 & 44,44 \\
\hline Pontificia Comillas & 62,50 & 50,00 \\
\hline Pontificia de Salamanca & 28,57 & 50,00 \\
\hline Pública de Navarra & 25,00 & 12,50 \\
\hline Ramón Llull & 37,50 & 50,00 \\
\hline Rey Juan Carlos & 55,56 & 28,57 \\
\hline Rovira i Virgili & 16,67 & 28,57 \\
\hline San Pablo - CEU & 16,67 & - \\
\hline
\end{tabular}




\section{ANEXO III}

Otros indicadores (no WCAG, no 508) por universidades

\begin{tabular}{|c|c|c|c|c|}
\hline & \multirow{2}{*}{\multicolumn{2}{|c|}{ Páginas institucionales }} & & \\
\hline & & & \multicolumn{2}{|c|}{ Páginas planes de estudios } \\
\hline Universidad & $\begin{array}{c}\% \\
\text { cumplimiento } \\
\text { indicadores } \\
\text { WCAG } 2006\end{array}$ & $\begin{array}{c}\% \\
\text { cumplimiento } \\
\text { indicadores } \\
\text { WCAG 2001 }\end{array}$ & $\begin{array}{c}\% \\
\text { cumplimiento } \\
\text { indicadores } \\
\text { WCAG } 2006\end{array}$ & $\begin{array}{c}\% \\
\text { cumplimiento } \\
\text { indicadores } \\
\text { WCAG } 2001\end{array}$ \\
\hline Alfonso X el Sabio & 40,00 & 50,00 & 50,00 & 50,00 \\
\hline Antonio de Nebrija & 33,33 & 83,33 & 0,00 & 66,67 \\
\hline Autónoma de Barcelona & 66,67 & 66,67 & 75,00 & 66,67 \\
\hline Autónoma de Madrid & 33,33 & 66,67 & 50,00 & 66,67 \\
\hline Camilo José Cela & 50,00 & 50,00 & 66,67 & 25,00 \\
\hline Cardenal Herrera - CEU & 75,00 & 100,00 & 0,00 & - \\
\hline Carlos III de Madrid & 50,00 & 100,00 & 66,67 & 66,67 \\
\hline Católica de Avila & 40,00 & 50,00 & 33,33 & 40,00 \\
\hline Católica San Antonio & 50,00 & 66,67 & 0,00 & 66,67 \\
\hline Complutense de Madrid & 100,00 & 75,00 & 100,00 & 50,00 \\
\hline A Coruña & 75,00 & 50,00 & 66,67 & 50,00 \\
\hline Alcalá & 66,67 & 100,00 & 0,00 & 66,67 \\
\hline Alicante & 100,00 & 100,00 & 100,00 & 33,33 \\
\hline Almería & 37,50 & 100,00 & 33,33 & 66,67 \\
\hline Barcelona & 75,00 & 50,00 & 33,33 & 100,00 \\
\hline Burgos & 50,00 & 66,67 & 50,00 & 66,67 \\
\hline Cádiz & 75,00 & 83,33 & 50,00 & 50,00 \\
\hline Cantabria & 33,33 & 50,00 & 33,33 & 66,67 \\
\hline Castilla-La Mancha & 75,00 & 40,00 & 0,00 & 50,00 \\
\hline Córdoba & 66,67 & 75,00 & 50,00 & 66,67 \\
\hline Deusto & 75,00 & 50,00 & 33,33 & 66,67 \\
\hline Extremadura & 66,67 & 50,00 & 66,67 & 50,00 \\
\hline Girona & 25,00 & 66,67 & 33,33 & 33,33 \\
\hline Granada & 100,00 & 50,00 & 50,00 & 66,67 \\
\hline Huelva & 25,00 & 66,67 & 50,00 & 66,67 \\
\hline Illes Balears & 33,33 & 50,00 & 25,00 & 50,00 \\
\hline Jaén & 50,00 & 40,00 & 25,00 & - \\
\hline La Laguna & 20,00 & 66,67 & 50,00 & 66,67 \\
\hline La Rioja & 66,67 & 66,67 & 25,00 & 66,67 \\
\hline
\end{tabular}




\section{ANEXO III (continuación)}

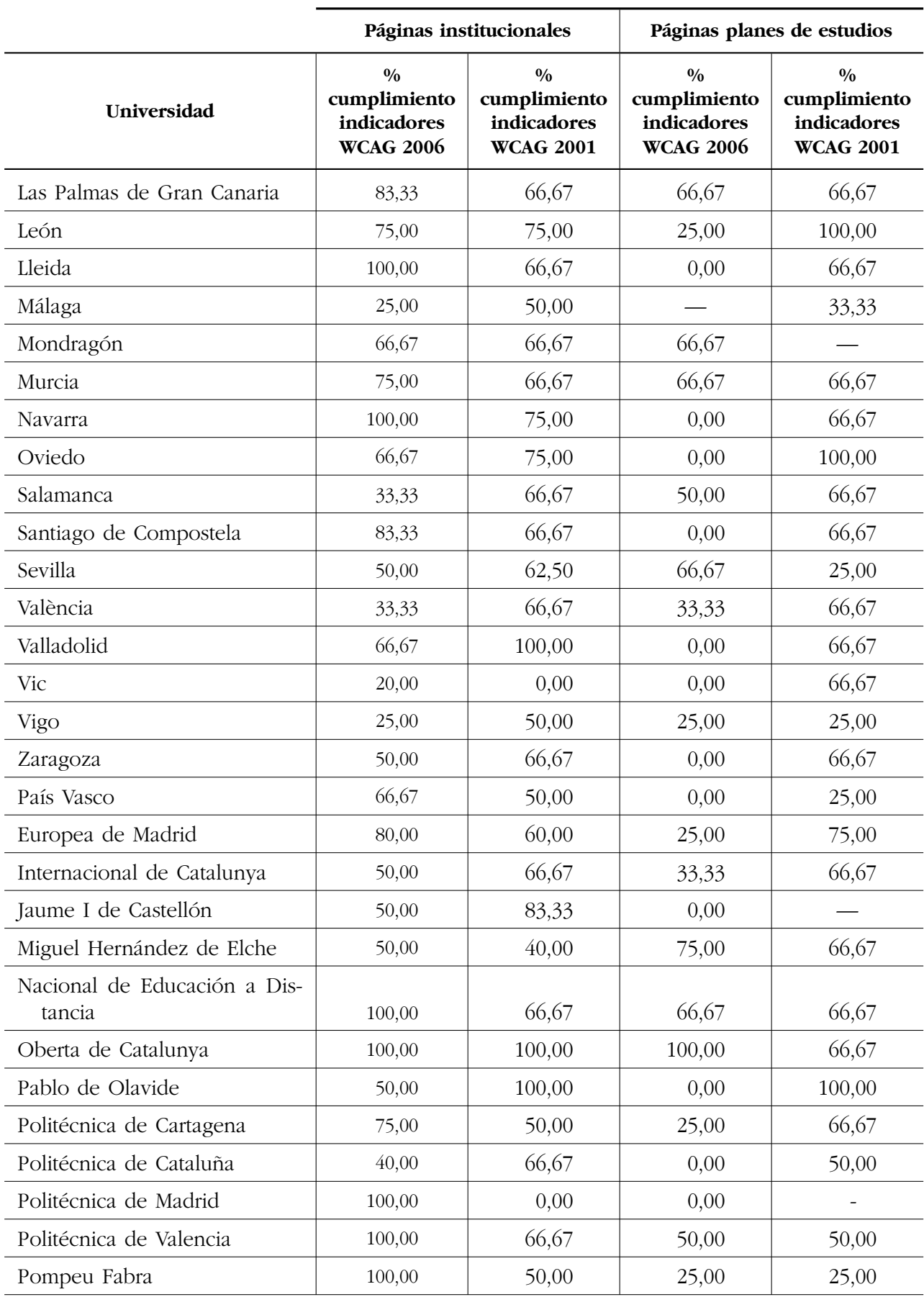




\section{ANEXO III (continuación)}

\begin{tabular}{l|c|c|c|c}
\cline { 2 - 5 } \multicolumn{1}{c}{ Universidad } & $\begin{array}{c}\text { Páginas institucionales } \\
\text { cumplimiento } \\
\text { indicadores } \\
\text { WCAG 2006 }\end{array}$ & $\begin{array}{c}\text { \% } \\
\text { cumplimiento } \\
\text { indicadores } \\
\text { WCAG 2001 }\end{array}$ & $\begin{array}{c}\text { Páginas planes de estudios } \\
\text { cumplimiento } \\
\text { indicadores } \\
\text { WCAG 2006 }\end{array}$ & $\begin{array}{c}\text { \% } \\
\text { cumplimiento } \\
\text { indicadores } \\
\text { WCAG 2001 }\end{array}$ \\
\hline Pontificia Comillas & 100,00 & 66,67 & 75,00 & 25,00 \\
\hline Pontificia de Salamanca & 40,00 & 33,33 & 0,00 & 50,00 \\
\hline Pública de Navarra & 66,67 & 50,00 & 33,33 & - \\
\hline Ramón Llull & 50,00 & 50,00 & 100,00 & 100,00 \\
\hline Rey Juan Carlos & 60,00 & 60,00 & 25,00 & 20,00 \\
\hline Rovira i Virgili & 0,00 & 100,00 & 0,00 & 25,00 \\
\hline San Pablo - CEU & 20,00 & 50,00 & - & 50,00 \\
\hline
\end{tabular}

